\title{
The effects of maternal immunity and age structure on population immunity to measles
}

\author{
A. McKee • M. J. Ferrari • K. Shea
}

Received: 22 July 2014 / Accepted: 29 December 2014 / Published online: 25 January 2015

(C) Springer Science+Business Media Dordrecht 2015

\begin{abstract}
Measles was successfully eradicated in the PanAmerican Health Region in 2002. However, maintenance of elimination in parts of Africa, Europe, the USA, and other regions is proving difficult, despite apparently high vaccine coverage. This may be due to the different age structure in developed and developing populations, as well as to differences in the duration of maternal immunity. We explore the interaction between maternal immunity and age structure and quantify the resulting immunity gap between vaccine coverage and population immunity; we use this immunity gap as a novel metric of vaccine program success as it highlights the difference between actual and estimated immunity. We find that, for some combinations of maternal immunity and age structure, the accepted herd immunity threshold is not maintainable with a singledose vaccine strategy for any combination of target age and coverage. In all cases, the herd immunity threshold is more difficult to maintain in a population with developing age structure. True population immunity is always improved if the target age at vaccination is chosen for the specific combination of maternal immunity and age structure.
\end{abstract}

Electronic supplementary material The online version of this article (doi:10.1007/s12080-014-0250-8) contains supplementary material, which is available to authorized users.

A. McKee $(\bowtie) \cdot$ K. Shea

Department of Biology, Pennsylvania State University, 208 Mueller Laboratory, University Park, PA 16802, USA

e-mail: azm200@psu.edu

M. J. Ferrari

Department of Biology, Pennsylvania State University, W243

Millennium Science Complex, University Park, PA 16802, USA
Keywords Context-dependent management $\cdot$ Disease elimination $\cdot$ Maternal immunity $\cdot$ Measles $\cdot$ Vaccination

\section{Introduction}

Great progress has been made towards worldwide measles eradication, yet it still remains an elusive objective, as endemic disease persists in some places, and is reintroduced to others where it was long absent. One aspect of the measles problem is maintenance of elimination; the disease has begun to reemerge in places where it was thought to be eliminated. Measles was officially eliminated from the USA in 2000 and the Pan-American Health region (PAHO region) in 2002 (Castillo-Solórzano et al. 2011a, b), and, despite recent outbreaks, endemic transmission has not reemerged. However, other countries have not been as successful at maintaining elimination. Europe has seen recent increases in transmission, despite promising improvements in the early 2000s (Morbidy and Mortality Weekly Report 2011), including places like Germany (Roggendorf et al. 2010; van Treeck 2006) and France (Parent du Châtelet et al. 2010). Recent outbreaks have occurred in parts of southern Africa where measles was previously reduced near the point of elimination (Shibeshi et al. 2014). Additionally, despite the disease officially remaining eliminated in the USA, there have been recent outbreaks that cast doubt on the actual population immunity to measles in the USA (Parker and Staggs 2006; Sugerman et al. 2010).

With measles, as with any other directly transmissible, immunizing infection, there is some threshold level of immunity in the population, called the herd immunity threshold, beyond which the disease cannot invade (Anderson and May 1991). The goal of measles vaccination programs is to achieve and maintain a sufficiently large immunized population that this 
threshold will be met, and measles will be locally eradicated and unable to reinvade. The conventional wisdom for measles is that this threshold level of immunity is 90-95\% (Hall and Jolley 2011; Moss and Griffin 2006).

Measles vaccination programs since the 2002 PAHO elimination are typically composed of multiple strategies for vaccine distribution (Danet and Fermon 2013; Koehlmoos et al. 2011). Here, we consider routine immunizations (RIs), in which children of specific ages are vaccinated during clinic visits (Bauch et al. 2009). RIs, in combination with other types of vaccination campaigns, were used to effectively eradicate measles in the Americas by 2002 (Castillo-Solorzano et al. 2011a, b). RIs are targeted at a specific age; the World Health Organization recommends measles vaccination between 9 and 12 months of age (WHO 2012).

Trans-placentally acquired maternal immunity temporarily protects infants born to immune mothers, but interferes with vaccine efficacy (Cutts et al. 1995; Gans et al. 2001). Vaccines administered before this maternal immunity wanes will be ineffective, but otherwise, there is minimal disadvantage to vaccinating children as early as possible. Therefore, there is a window of susceptibility between the waning of maternal immunity and the average age of infection during which vaccination is likely to be effective and prophylactic in most children (McLean and Anderson 1988a, b). The optimal age of vaccination will fall within this age window, where the lower end is determined by acquired maternal immunity (Moss and Griffin 2006; McLean and Anderson 1988a, b), and the upper end is classically considered to be determined by local disease incidence (McLean and Anderson 1988a, b).

There is uncertainty in the literature about when, on average, maternal immunity wanes in any specific population. Some evidence suggests that vaccine-derived maternal immunity wanes earlier than naturally derived maternal immunity (that is, maternal immunity from women who have been infected with measles) (Leuridan et al. 2010), but the magnitude of this difference and the effect it may have on the optimal age at which to vaccinate is unclear. Additionally, the mother's health (Scott et al. 2005) as well as local nutrition and breast feeding practices have been shown to affect the rate of waning of maternal immunity and are difficult to know precisely (Cáceres et al. 2000). What limited evidence we have available suggests that the rate at which maternal immunity wanes varies from country to country (McLean and Anderson 1988a, b).

In places where measles is endemic, children may become infected and therefore become naturally resistant before the age at first vaccination. Thus, vaccines administered too late fail to prevent disease and may be considered as "wasted" doses - doses administered to a person already immune. It is classically understood that, as disease incidence declines, average age of infection increases (Roggendorf et al. 2010), thus increasing the upper age limit on the window of infant susceptibility. In these settings, a common policy is to increase the target age of routine immunizations so that a greater proportion of infants will have lost maternal immunity, and each dose is more likely to be more effective (Christie and Gay 2011).

However, we have seen unexpected outbreaks in countries with what was thought to be good measles control, such as the 2009 epidemic in Burkina Faso (Kidd et al. 2012) and the 2010 epidemic in Malawi (Minetti et al. 2013a, b). These countries had otherwise low disease incidence and high routine coverage. However, these unexpected epidemics indicate that, despite the low measles incidence, there was a substantial susceptible pool. The interaction between vaccine effectiveness, maternal immunity, and the chosen age target for routine immunization may have contributed to the rapid build-up of this susceptible pool and the underestimation of outbreak risk.

Differences in age structure could also contribute to observed differences in population immunity resulting from similarly targeted RIs. Notably, the proportion of the population in the Americas that is $<1$ year old is much smaller than the proportion of the African population that is $<1$ year old. As such, the number of children in the susceptible window in Africa will make up a relatively large proportion of the population and may suggest the need for a different target age for routine immunization. Consequently, the upper end of the infant susceptibility window may also be determined by the age structure of the region, rather than solely by disease incidence. Importantly, if the target age of vaccination is not tailored to average local immunity and age structure, the proportion of infants no longer maternally immune but not yet vaccinated might be too large for herd immunity to be achievable (Fig. 1a). In essence, if more than $5 \%$ of the population is in the susceptible window between waning of maternal immunity and vaccination, a $95 \%$ population immunity level will be unachievable.

The existence of this window of susceptibility (Fig. 1) means that population immunity will always be less than or equal to routine immunization coverage in disease-free settings. That is, some doses are administered to infants who still retain their maternal antibodies, and some susceptible infants are not yet eligible for the vaccination. Thus, for any combination of age target, age distribution, and waning rate, we can characterize the immunity gap between coverage and population immunity; this immunity gap tells us the amount by which population immunity falls short of coverage.

In this paper, we develop a discrete-time age-structured population model for the distribution of immunity in a disease-free population and analyze the equilibrial states of this model. We analyze the size and distribution of the crosshatched regions in Fig. 1, which show the proportion of the population not covered by either maternal immunity or vaccination. We use this analysis to assess the relative size and age distribution of the susceptible population resulting from a variety of vaccine plans, specified by target age and coverage, and the effect of these plans on population immunity. We also 
Fig. 1 Two sample age distributions of immunity within a population. The dark gray region is the portion still maternally immune, the pale gray region is the vaccinated proportion, and the hatched region is the proportion that remains susceptible. The $x$ axis is shown on a log scale, so the total hatched regions in $\mathbf{a}$ and $\mathbf{b}$ are of similar area. In $\mathbf{a}$, the vaccine is administered too late, and too many infants remain susceptible. In $\mathbf{b}$, the vaccine is administered too early, so too few doses are effective. Both of these figures use a developing population age structure assuming 6-month maternal immunity
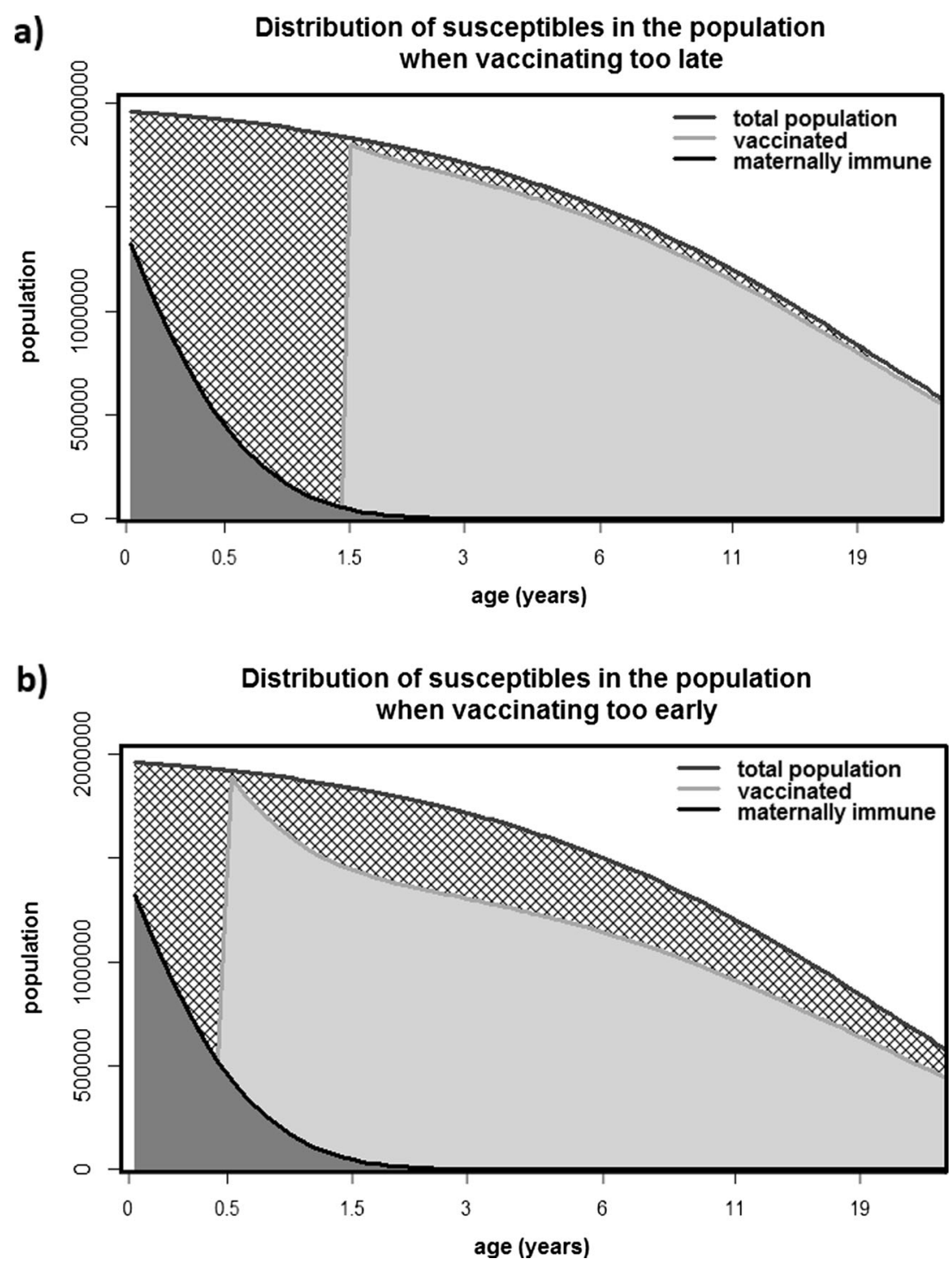

calculate the immunity gap between population immunity and coverage for each of these vaccine plans. We then evaluate these vaccine plans (specific combinations of target vaccination age and planned coverage) for a range of age distributions to quantify the impact of age structure on vaccine program success. We then suggest some methods for selecting among a range of vaccine plans where herd immunity is equally achievable or unachievable.

\section{Materials and methods}

We developed an age-structured model for a human population, using 131 age classes. Age classes are monthly, up to 5 years old, and then are yearly until 75 years old, when we assume individuals are removed from the population. The relative size of these age classes is determined by a specified age structure. We explored two base age structures - a concave one, representing an idealized developed population, as in the USA, and a convex one, representing an idealized developing population, as in Sub-Saharan Africa (see Fig. S1 in Supplemental Material). Throughout this paper, we use "developing" and "developed" to refer solely to the age structure of the region, not their economic development. We also consider the effect of a range of intermediate age structures, based on a weighted $(\alpha)$ average of these two base age structures.

Each of these age classes are then divided into one of three immune classes - maternally immune, susceptible, or vaccinated. In this study, we omit the naturally immune classes, as we conduct a steady-state analysis under the assumption of long-term disease absence, as might be the case in most of the USA. We then quantify the relative size and age distribution of these classes, paying special attention to the susceptible class, as that is the class of interest for population immunity; the 
susceptible class can make up no more than $5 \%$ of the population if we are to maintain herd immunity.

To divide these age classes into the immune classes, we first calculate the proportion of individuals who are born with maternal immunity (Eq. 1). Then, accounting for maternal immunity waning at a specified rate, we calculate the proportion of individuals in each older age class. Once we know what proportion of individuals in each age class is maternally immune, we can calculate effective vaccine cover from a specified target age and coverage, given that maternally immune individuals cannot be successfully immunized. From there, we assume that the proportion of vaccinated individuals in each subsequent age class remains the same. We assume that the remainder of the population (that is, those not maternally immune or vaccinated) is susceptible.

Vaccines are administered at a given age, $t$, with a given coverage, $C$; this combination of specifications is termed the vaccine plan. The proportion that are successfully immunized is a proportion equal to the coverage, $C$, of the proportion of individuals that are not maternally immune in the target age class, $t$. We assume that these two proportions are independent, as a proportion of the vaccinated population is still maternally immune and so is vaccinated but does not achieve vaccine-derived immunity — that is, is not considered successfully vaccinated. Each subsequent age class has the same proportion of successfully vaccinated individuals as the first vaccinated age class, $t$, but loses some immune individuals as maternal immunity continues to wane.

The probability that any individual is born with maternal immunity depends on the equilibrium state of a generational model. The probability that an individual is born maternally immune in generation $T, p_{M T}$, is equal to the probability that his/her mother was successfully vaccinated in the previous generation, $V_{T-1}$. This, in turn, is dependent on the vaccine coverage, $C$, and the probability that the mother was no longer maternally immune when the vaccine was administered at age $t$, so that:

$p_{M T}=V_{T-1}=C\left[1-\left(\frac{\omega-1}{\omega}\right)^{t} p_{M(T-1)}\right]$

Here, $\omega$ is the average age where maternal immunity wanes. Assuming that there has been no change in vaccine policy or maternal immunity between generations, the steadystate proportion of infants born with maternal immunity, $p_{M^{*}}$ (where $p_{M^{*}}=p_{M T}=p_{M(T-1)}$ ), is found by solving the resulting equation:

$p_{M^{*}}=C\left[1-\left(\frac{\omega-1}{\omega}\right)^{t} p_{M^{*}}\right]$

We find the steady-state proportion of infants born with maternal immunity is:

$$
\frac{C}{1+C\left(\frac{\omega-1}{\omega}\right)^{t}}
$$

Notably, this proportion goes up with coverage, but saturates at some level dependent on the average age of maternal immunity waning and the age at vaccination.

For each successive age class, we then find the proportion of individuals that remain maternally immune, based on one of two exponential decay functions with an average age of waning of either 3 or 6 months. We chose these average ages because most estimates in the literature fall within that range (Cáceres et al. 2000; Waaijenborg et al. 2013). That is, each successive age class has $\frac{\omega-1}{\omega}$ times as many maternally immune individuals as the previous class, where $\omega$ is either 3 or 6 (see Fig. S2 in Supplemental Material). Maternal immunity continues to wane in this way until the 5-year age class, when we assume that no individuals are maternally immune any longer.

The proportion of each age class left susceptible is simply the proportion neither maternally immune nor immunized. From this model, we have an age distribution of susceptibility, with different numbers of susceptible individuals in each age class. We first calculate the total proportion immune achieved by any specific vaccine plan given by a target age and coverage. We then examine the resulting age distribution of susceptibility, and how that varies with coverage. We also examine the immunity gap between programmatic vaccine coverage and achieved population immunity, with the idea that this immunity gap might provide a means of discriminating between apparently equivalent vaccine plans. Finally, we assess the dependence of this vaccine program success on age structure, both in how age structure can affect the recommended vaccine plan and how the success of a specific vaccine plan depends significantly on age structure alone.

\section{Results}

We first develop contours for the maintainable population immunity for a given age structure and maternal immunity duration, under a specific vaccine plan comprising of a specified target age (in months) and coverage (Fig. 2). For a developing age structure with maternal immunity waning at 6 months, the commonly quoted herd immunity threshold of $95 \%$ is unachievable, no matter when children are vaccinated and what coverage is achieved (Fig. 2a). However, if vaccination is targeted at the older age classes $(12+$ months $)$ and achieves high coverage, then over $90 \%$ of the population will be immune. When maternal immunity in a developing age structure is assumed to wane at 3 months, the herd immunity threshold is achievable, but only for extraordinarily high coverage and over a limited age range for vaccination (Fig. 2b).

In comparison, it is relatively easy to achieve the herd immunity threshold with a developed age structure. With maternal immunity waning at 6 months, the herd immunity threshold is achievable if vaccination occurs at an older age and with 


\section{a) Population immunity maintained in a developing population with 6 month maternal immunity}

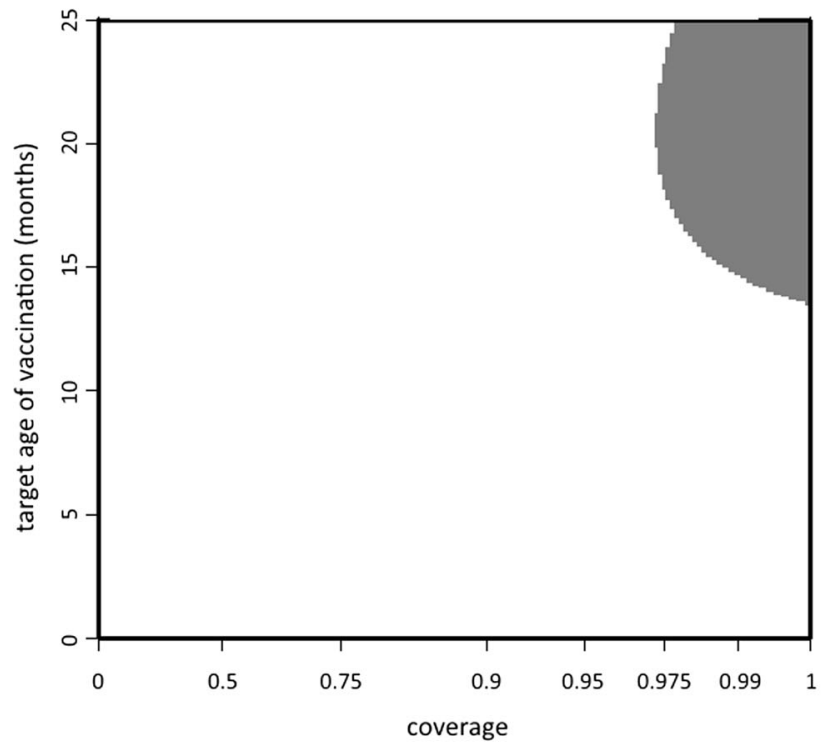

\section{c) Population immunity maintained in a developed population with 6 month maternal immunity}

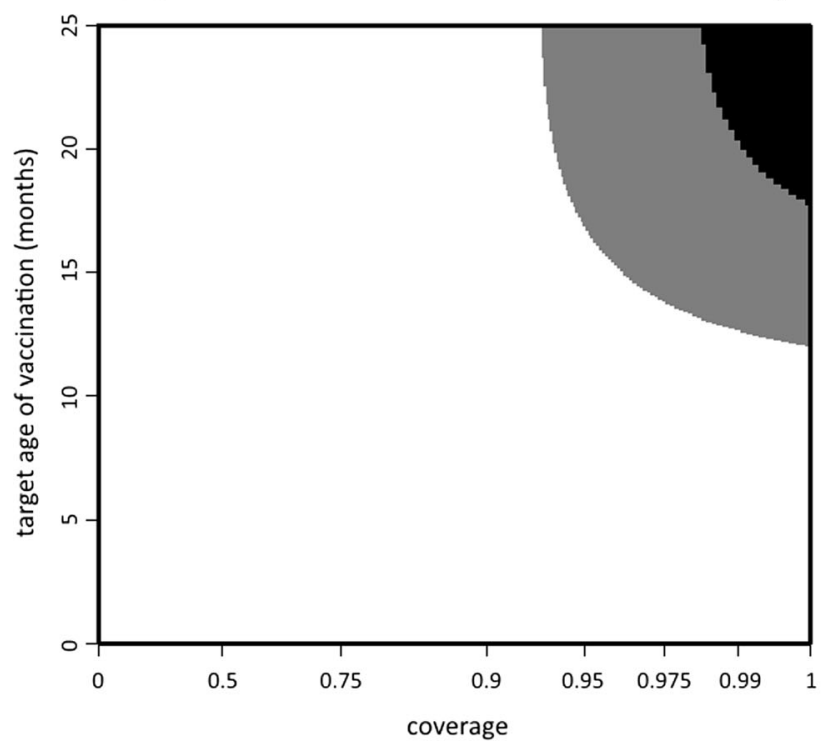

Fig. 2 a, b Maintainable population immunity for maternal immunity waning at 6 and 3 months, respectively, in a region with a developing age structure. c, d Maintainable population immunity for maternal immunity

high coverage (Fig. 2c). Notably, this successful age range is strictly older than the age range successful in a developing region with maternal immunity waning at 3 months (Fig. 2b). If maternal immunity wanes at 3 months on average, then the herd immunity threshold is achievable with high coverage at a wide range of ages (Fig. 2d). Taking the difference between the two developed surfaces (Fig. 3a) shows that the average age at which maternal immunity wanes makes the largest difference in population immunity when vaccination happens at a young age and with high coverage. The general

\section{b) Population immunity maintained in a developing population with $\mathbf{3}$ month maternal immunity}



\section{d) Population immunity maintained in a developed population with $\mathbf{3}$ month maternal immunity}

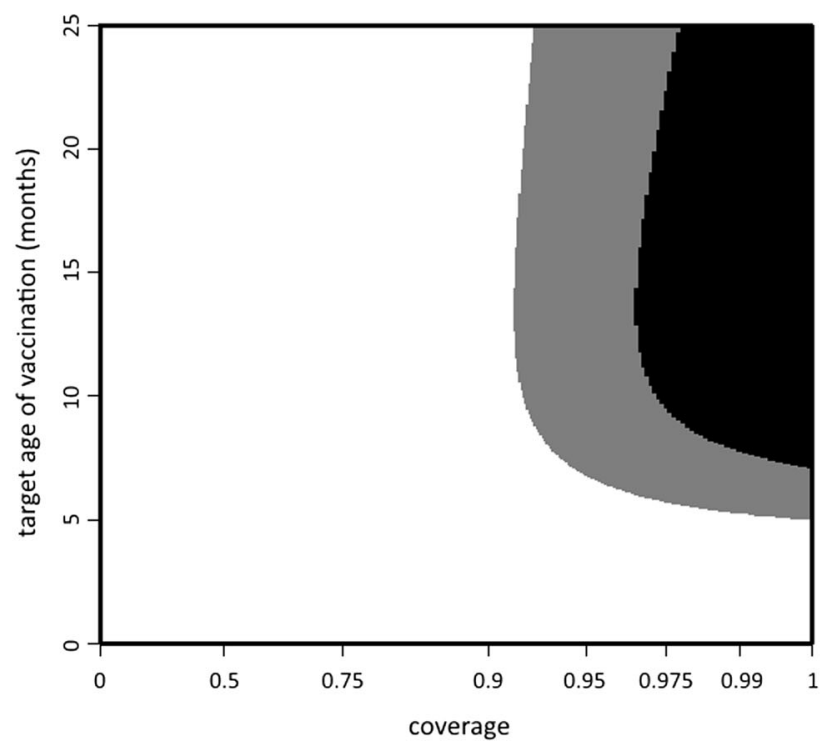

waning at 6 and 3 months, respectively, in a region with developed age structure. Strategies that meet the $95 \%$ threshold are shown in black; those that meet the $90 \%$ threshold are shown in gray

shape of this difference in response to waning maternal immunity is the same regardless of the underlying age structure. Comparing the results from the developed and the developing age structures shows that the shape of the differences due to underlying population age structure (Fig. 3b) is similar no matter the rate at which maternal immunity wanes, although the maximum magnitude of the difference varies (the shape of the immunity gap between coverage and population immunity is not the same, as shown in Fig. S4 in Supplemental Material). 
Fig. 3 a The difference in population immunity due to differences in maternal immunity (i.e., the difference between Fig. 2c and d). b Difference in population immunity for maternal immunity waning at 3 months between regions with a developed and developing age structure (i.e., the difference between Fig. $2 b$ and d). Note that the target ages of vaccination which minimize the difference due to a difference in maternal immunity are where the difference due to a difference in age structure starts to increase

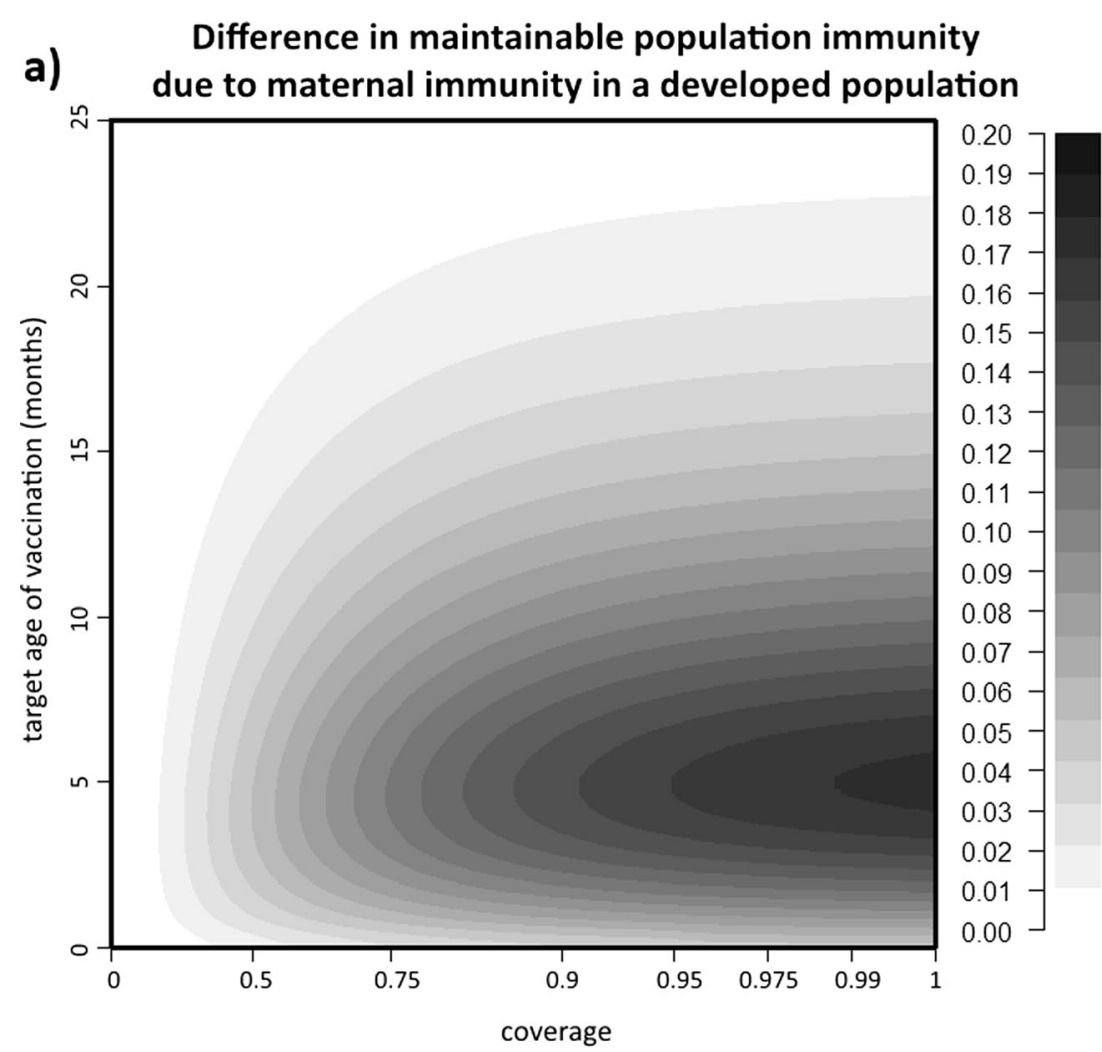

b) Difference in maintainable population immunity

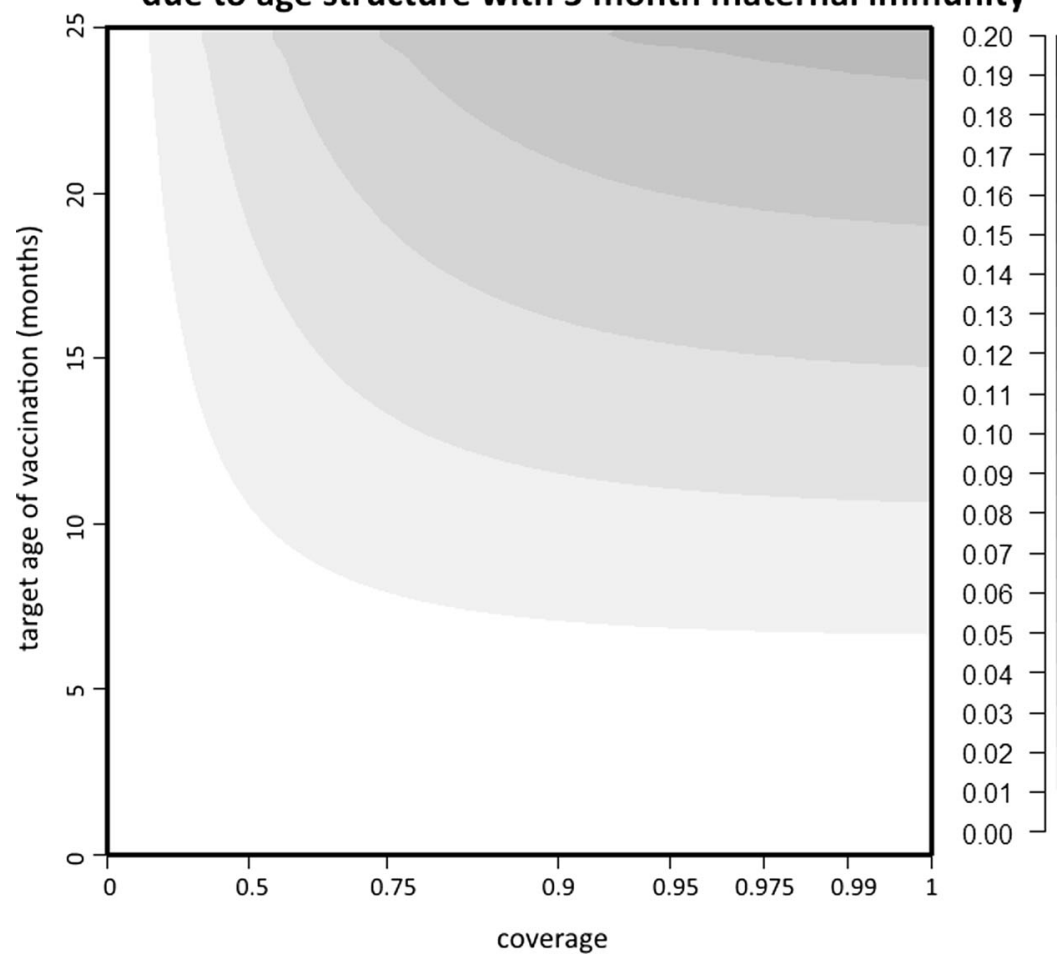

We quantify the impact of age structure on vaccine program success by considering a range of age structures, which are linear composites of the developing and developed age structures according to some weight, $\alpha$. We use $\alpha$ to represent the proportional weight of the developing age structure-for example, when $\alpha$ is 0.25 , this means that we took a weighted 
average of the two age structures, with a $25 \%$ weight on the developing age structure and a $75 \%$ weight on the developed age structure. When $\alpha$ is 0 , the age structure is precisely the developed age structure, and when $\alpha$ is 1 , the age structure is precisely the developing age structure. We find that, even when vaccinating to achieve maximum population immunity given a specific age structure (Fig. 4a), the achieved population immunity continually decreases as the age structure becomes more and more similar to the purely developing one. With maternal immunity waning at 6 months, the $95 \%$ threshold is entirely unachievable once more than $60 \%$ of the age structure is of the developing form. The situation is even worse if the same vaccine plan (i.e., same coverage and target age) is applied across all age structures, especially if the one that works best for the developing age structure is chosen (Fig. 4b).

Population immunity is always less than vaccine coverage (to see how they relate, see Fig. S3 in Supplemental Material), but the difference depends on the specific vaccine policy and population structure. We show the immunity gap for several target ages, which are sufficient for herd immunity in a developing region, where the target age that minimizes the immunity gap changes depending on vaccine coverage (Fig. 5). Notably, the shape of these different curves changes depending on the target age of vaccination, as the source of the immunity gap (age structure or maternal immunity) changes depending on target age. This change in shape means that the target age that minimizes the immunity gap changes with respect to coverage. In Fig. 5a, for coverages below around $67 \%$ (indicated by the lighter vertical line), it is best (in terms of minimizing the immunity gap) to vaccinate at 10 months of age, as low coverage will mean low levels of maternal immunity. For higher coverages, it is best to vaccinate at 12 months. At around $92 \%$ coverage - indicated by the darker vertical line in Fig. 5a and the only vertical line in Fig. 5b-the formerly optimal target age becomes dramatically worse due to

a)

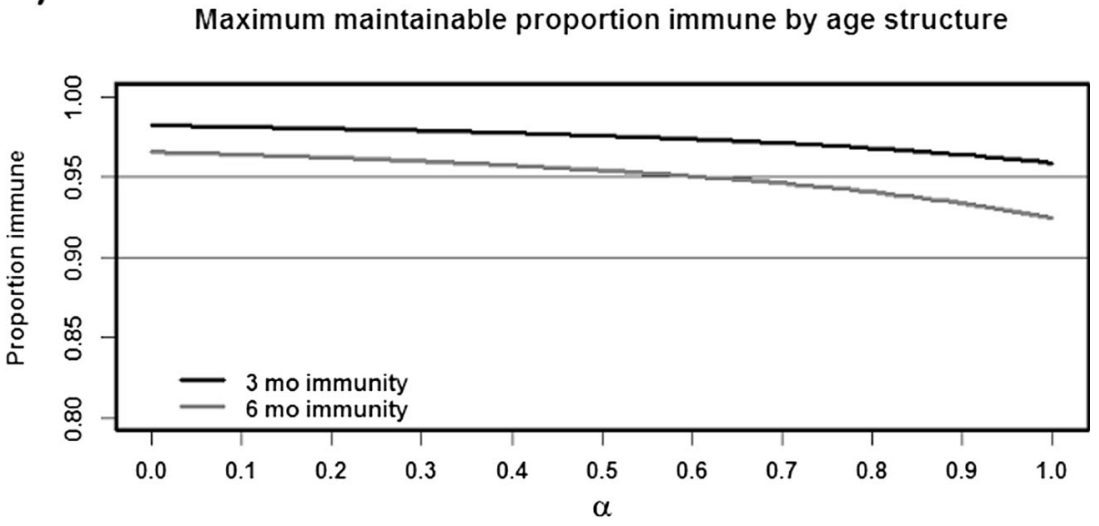

b)

Proportion immune by age structure when vaccinating at 12 months with $98 \%$ coverage

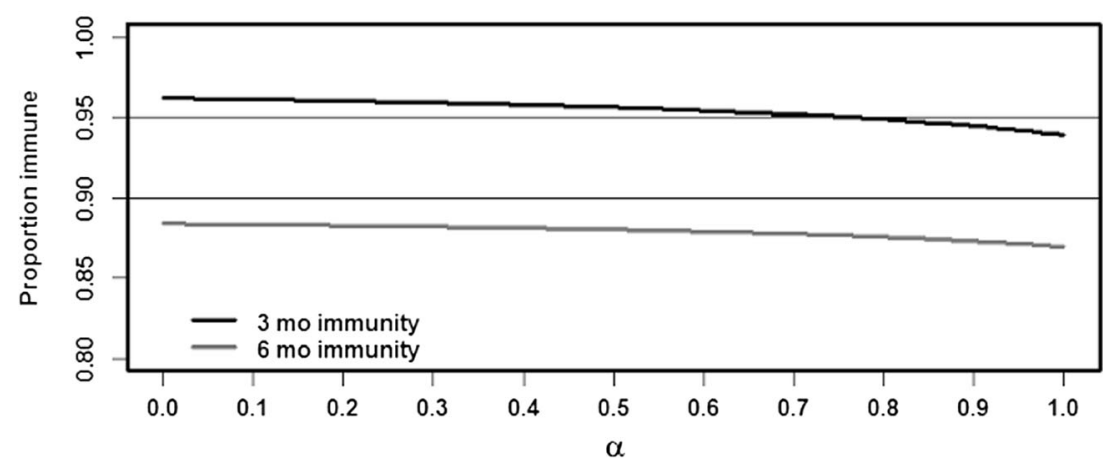

Fig. 4 The maintainable population immunity for a range of age structures. When $\alpha$ is 0 , the age structure is the idealized developed one. When $\alpha$ is 1 , the age structure is the idealized developing one. a As the age structure shifts from developed to developing, the maximum maintainable proportion immune continuously declines. Notably, the proportion immune for long maternal immunity is lower than that for short maternal immunity despite both being the specific maxima for that maternal immunity function. That is, the optimal vaccine policy for long maternal immunity is always less successful than the optimal for short maternal immunity. b The achievable proportion immune when vaccinating at 12 months. With a developed age structure and short maternal immunity, this is a successful vaccine policy. However, as the age structure shifts towards the purely developing one, the success of the vaccine policy continually declines. With long maternal immunity, maintainable immunity is similarly low everywhere, but still worse on a developing age structure 

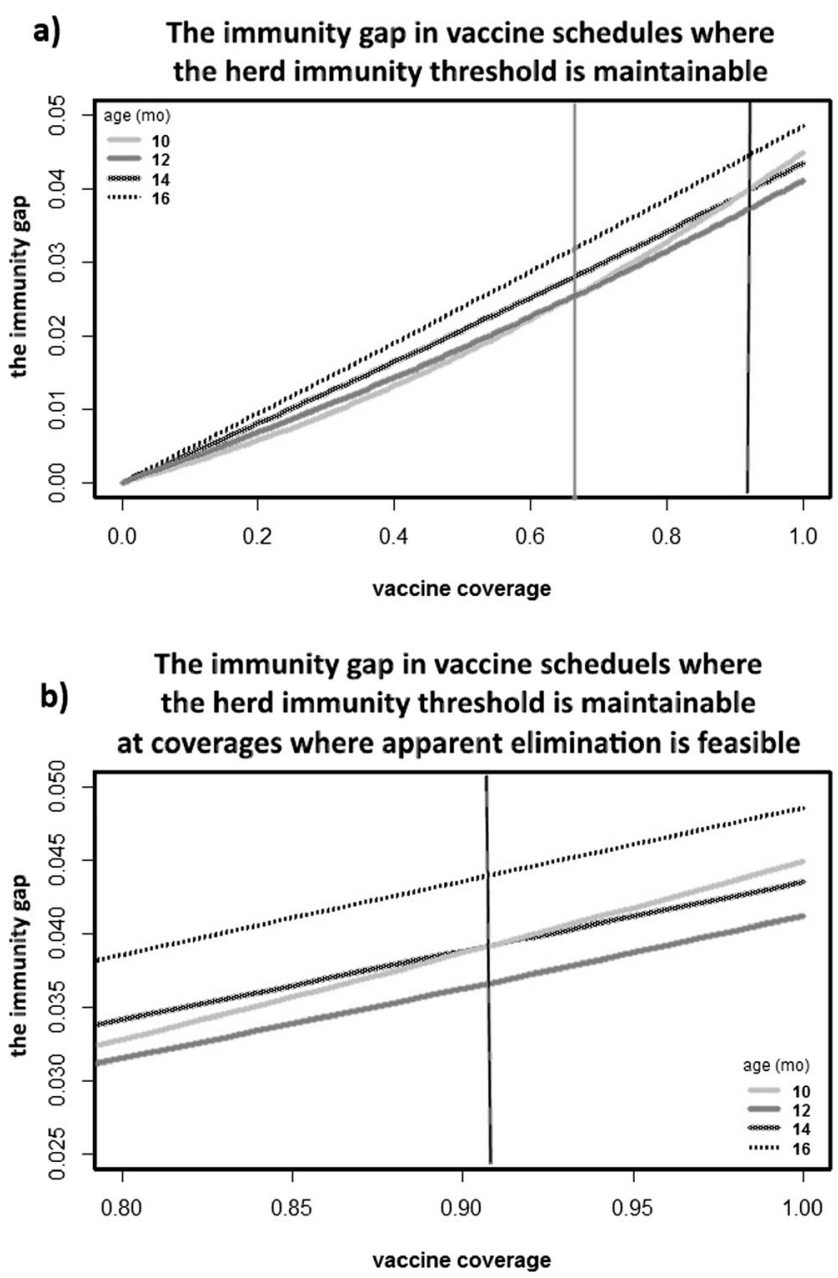

Fig. 5 These panels show the immunity gap - that is, the difference between vaccine coverage and population immunity - for a variety of target ages and coverages. Here, we are comparing these vaccine schedules in a developing population with 3-month maternal immunity. a At high coverage, all of these vaccine schedules could achieve herd immunity. However, as maternal immunity is less significant at older ages, it has a different effect on the immunity gap at the different ages. Notably, the immunity gap line when vaccinating at the youngest age with high coverage is significantly curved. b Focusing on the immunity gap at coverages that might be maintained in regions with measles elimination, it is clear that 12 months is the best age to vaccinate at all of these coverages in a region with this age structure and maternal immunity decay. However, whether it would be better to miss the target age by vaccinating a little too young (10 months) or a little too old (14 months) depends on coverage, with vaccinating younger ages still being worse at high coverages

the nonlinear interaction with maternal immunity, with the immunity gap exceeding that of all but the very oldest target ages.

\section{Discussion}

Our work shows that herd immunity may not be maintainable with a single-dose routine vaccine plan, even administered optimally, depending on the specific age structure and maternal immunity of the region. Fortunately, strategies for the maintenance of measles elimination commonly include a second dose. However, even in the context of a second-dose vaccine plan, it does not make sense to administer the first dose of a two-dose vaccine strategy ineffectively, with the hope that the second dose will make up for failings in the first, especially if the result is a large susceptible population that is too young to be caught by a second-dose vaccine strategy at all. Optimizing the first dose is beneficial in all cases. Our results suggest ways that we can maximize the impact of the first dose to improve population immunity by specifically targeting a vaccine program to the local context, specifically the demography and maternal immunity levels of the target population.

In principle, herd immunity is maintainable in all cases except when vaccinating in a population with a developing age structure and long maternal immunity (Fig. 2a). However, herd immunity is maintainable for a wider range of coverage and ages when vaccinating given a developed age structure (Fig. 2c,d). Notably, the range of target ages where herd immunity is maintainable in a developed region is much older than the age range where herd immunity is maintainable in a developing region, if it is maintainable at all in a developing region.

With a developing age structure, where the average age is fairly young, more children fall below the age of first vaccination, and herd immunity is more difficult to maintain. It is especially difficult if the choice of vaccine plan is based on the recommended strategy for a developed age structure, where the average age is much older (Fig. 4b). This demonstrates the importance of context-dependent vaccination planning. Population immunity improves when a vaccine plan optimized to the local age structure and maternal immunity function is chosen (Fig. 4a), so that the difficulty imposed by the age structure is effectively minimized. Interestingly, the age at vaccination that maximizes population immunity in a developing region is often younger than that which maximizes immunity in a developed region. That is, the luxury to vaccinate older individuals to minimize doses rendered ineffective by maternal immunity is affordable when vaccinating in a developed region, but age structure forces the vaccination of younger individuals when vaccinating in a developing region. Notably, reducing the age of first vaccination is a strategy not generally considered when making and implementing current vaccine policy.

Context-dependent vaccination planning may also be useful at a finer spatial scale. Vaccine program success in Africa is relatively patchy, with some countries having only sporadic outbreaks that suggest they are near elimination, while others still have regular seasonal outbreaks (Minetti et al. 2013a, b; Ferrari et al. 2008). These regional differences in vaccine program success may be due to differences in logistic effectiveness, but are likely also impacted by the interaction of maternal immunity and age structure. Just as regions with 
developing age structures, such as Africa, are different from regions with developed age structures, such as the Americas, different regions within Africa are different from each other with regard to age structure and also, potentially, the duration of maternal immunity. A context-dependent management strategy that takes into account local patterns in immunity and age structure and evaluates their relative significance is necessary in this case.

We introduce a new measure of program success called the immunity gap and examine the effect of changing target age or changing population age structure and maternal immunity on the relationship between coverage and the immunity gap. In some ways, the immunity gap represents "wasted" dosesthat is, the difference between population vaccine coverage and population immunity achieved. Regardless of vaccine plan or population, the immunity gap always increases as coverage increases. This is, in some ways, a numerical representation of the "last mile" problem (Klepac et al. 2013) - as measles control improves, the cost per case prevented increases. However, the way this the immunity gap scales with coverage varies depending on the source of the immunity gap.

If vaccines are administered too early (Fig. 5), the immunity gap varies nonlinearly (almost exponentially) with coverage. This is a signature of the maternal immunity function on population immunity. If the vaccine is administered later, or to a population with shorter maternal immunity, the immunity gap scales more linearly with coverage. By looking at these together, general recommendations can be made. If the source of this immunity gap is interference with maternal immunity, vaccines should be administered at older ages. If the source is the interaction with age structure, vaccines should be administered at younger ages. The immunity gap could provide a novel way to select between apparently equal vaccine plans. Choosing a vaccine plan to minimize the immunity gap given information about local age structure, maternal immunity levels, and achievable coverage would also maximize the proportional immunity conferred per dose administered.

The immunity gap is not the only means of selecting between vaccine plans. In cases where increasing the age of vaccination reduces the immunity gap, this change in age targets may increase the potential mortality associated with an epidemic by increasing the proportion of susceptible individuals who are very young. Targeting younger children for vaccination may increase the immunity gap nonlinearly, but could minimize loss of life, as a larger proportion of susceptible individuals would fall in the less at-risk age classes. When coverage is low, the immunity gap is small and the majority of susceptible individuals are in the oldest age classes. As coverage increases, the immunity gap increases and the proportion of susceptible individuals below the age of vaccination increases, although the absolute number may not. The magnitude of this shift depends on the target age of vaccination and the age distribution and maternal immunity function of the population. Again, these younger children are the individuals that are most likely to die during an epidemic (Wolfson et al. 2009).

An additional utility of the immunity gap measure (Fig. 5) is that using vaccine coverage as a proxy for population immunity may lead to a severe underestimation of disease risk, and the size of this underestimate is context dependent. This may explain some of the patterns in measles reemergence that we see in places with what was thought to be good vaccine coverage, such as Malawi and Germany. Malawi had reasonably good measles control, having seen a period of local elimination of disease. However, in 2010, there was a devastating outbreak, with the age distribution of cases being much older than expected (Minetti et al. 2013a, b). At the time, Malawi was officially administering a first dose of the measles vaccine to infants between the ages of 9 and 11 months, with a second dose comprising of a supplemental immunization campaign targeting individuals $<5$ years old. On the other hand, Germany has struggled to maintain herd immunity to measles for a while, with frequent outbreaks. The age distribution of some of these outbreaks (Siedler et al. 2011) is older than typically seen in a measles outbreak. In Germany, the first dose was classically administered at 12 months, with the second dose coming between 4 and 6 years of age. In 2001, Germany changed its guidelines, expanding the window for first dose administration and lowering the age of the second dose. In both cases, coverage was thought to be good, but outbreaks occurred. Thus, while administrative coverage may have been at, or above, the programmatic target, the combination of age distribution and waning of immunity may have resulted in subcritical immunity.

Our work does not include population heterogeneity, agespecific contact patterns, or age-specific force of infection. Such processes are certainly relevant for more complex dynamical analyses of measles incidence. We omit them, in part, because our work is an intentionally simple analysis to highlight the importance of an underconsidered tradeoff between age structure and maternal immunity. Additionally, singledose vaccine plans are not commonly implemented intentionally (Hall and Jolley 2011). There is evidence for the existence of remote locales with low disease incidence and low seconddose coverage (Minetti et al. 2013a, b), but most countries officially give a second dose of the measles vaccine, even though the second dose may not be administered at the recommended ages or at the ages with the largest remaining susceptible populations (Clark and Sanderson 2009). This research could provide a means to optimize vaccine policy for such locales, as well as other locales where second-dose coverage is patchy or otherwise uncertain, though future work incorporating a second dose would also be informative.

This work presents an equilibrial analysis of this disease system, meant to supplement existing dynamical work in an intuitive way. Future work could include additional aspects of 
vaccine program complexity, including target age ranges and a second-dose vaccine strategy, as well as disease dynamics. Nonetheless, this work has some interesting implications. As a larger proportion of any population is below the age of vaccination, herd immunity becomes increasingly difficult to maintain - that is, if a population has a relatively large infant population, herd immunity to measles will be difficult, if not impossible, to maintain with a single-dose vaccine plan. Population structure also interacts with the waning of maternal immunity in ways that can mislead us into thinking that population immunity is better than it really is. However, our work suggests the possibility of improving local disease control, despite obvious difficulties, by taking specific contextdependent factors into account (Hall and Jolley 2011). Our model could be adapted to fit any specified age structure and maternally derived immunity waning function and then used to make a recommendation that minimizes the immunity gap given an estimated coverage. These results are only complicated if maternal immunity lasts too long to effectively vaccinate any earlier. However, given a heavily youth-weighted age structure, vaccinating earlier may be beneficial, as a seconddose vaccine strategy will be better at catching those for whom the vaccine was not effective.

Acknowledgments We thank Ottar Bjørnstad, whose comments contributed to this manuscript. We acknowledge funding from EEID award 1 R01 GM105247-01 jointly funded through National Institutes of Health and National Science Foundation and from the Vaccine Modeling Initiative funded by the Bill and Melinda Gates Foundation.

\section{References}

Anderson RM, May RM (1991) Infectious diseases of humans: dynamics and control. Oxford University Press, Oxford

Bauch CT, Szusz E, Garrison LP (2009) Scheduling of measles vaccination in low-income countries: projections of a dynamic model. Vaccine 27:4090-4098. doi:10.1016/j.vaccine.2009.04.079

Cáceres VM, Strebel PM, Sutter RW (2000) Factors determining prevalence of maternal antibody to measles virus throughout infancy: a review. Clin Infect Dis 31:110-119. doi:10.1086/313926

Castillo-Solórzano C, Marsigli C, Danovaro-Holliday MC et al (2011a) Measles and rubella elimination initiatives in the Americas: lessons learned and best practices. J Infect Dis 204(Suppl):S279-S283. doi: 10.1093/infdis/jir216

Castillo-Solórzano C, Reef SE, Morice A et al (2011b) Guidelines for the documentation and verification of measles, rubella, and congenital rubella syndrome elimination in the region of the Americas. J Infect Dis 204(Suppl 2):S683-S689. doi:10.1093/infdis/jir471

Christie AS, Gay A (2011) The Measles Initiative: moving toward measles eradication. J Infect Dis 204:S14-S17. doi:10.1093/infdis/ jir075

Clark A, Sanderson C (2009) Timing of children's vaccinations in 45 low-income and middle-income countries: an analysis of survey data. Lancet 373:1543-1549. doi:10.1016/S0140-6736(09)60317-2

Cutts FT, Grabowsky M, Markowitz LE (1995) The effect of dose and strain of live attenuated measles vaccines on serological responses in young infants. Biologicals 23:95-106
Danet C, Fermon F (2013) Management of a measles epidemic: Practical guide for doctors, nurses, labratory technicians, medical auxiliaries and logisticians. Médecins Sans Frontières, Paris

Ferrari MJ, Grais RF, Bharti N et al (2008) The dynamics of measles in sub-Saharan Africa. Nature 451:679-684. doi:10.1038/nature06509

Gans H, Yasukawa L, Rinki M et al (2001) Immune responses to measles and mumps vaccination of infants at 6, 9, and 12 months. J Infect Dis 184:817-826. doi:10.1086/323346

Hall R, Jolley D (2011) International measles incidence and immunization coverage. J Infect Dis 204(Suppl):S158-S163. doi:10.1093/ infdis/jir124

Kidd S, Ouedraogo B, Kambire C et al (2012) Measles outbreak in Burkina Faso, 2009: a case-control study to determine risk factors and estimate vaccine effectiveness. Vaccine 30:5000-5008. doi:10. 1016/j.vaccine.2012.05.024

Klepac P, Metcalf CJE, McLean AR, Hampson K (2013) Towards the endgame and beyond: complexities and challenges for the elimination of infectious diseases. Philos Trans R Soc B Biol Sci. doi:10. 1098/rstb.2012.0137

Koehlmoos TP, Uddin J, Sarma H (2011) Impact of measles eradication activities on routine immunization services and health systems in Bangladesh. J Infect Dis 204(Suppl):S90-S97. doi:10.1093/infdis/ jir086

Leuridan E, Hens N, Hutse V et al (2010) Early waning of maternal measles antibodies in era of measles elimination: longitudinal study. Br Med J 340:c1626. doi:10.1136/bmj.c1626

McLean AR, Anderson RM (1988a) Measles in developing countries. Part I. Epidemiological parameters and patterns. Epidemiol Infect 100:111-133

McLean AR, Anderson RM (1988b) Measles in developing countries. Part II. The predicted impact of mass vaccination. Epidemiol Infect 100:419-442

Minetti A, Bopp C, Fermon F et al (2013a) Measles outbreak response immunization is context-specific: insight from the recent experience of médecins sans frontières. PLoS Med 10:e1001544. doi:10.1371/ journal.pmed.1001544

Minetti A, Kagoli M, Katsulukuta A et al (2013b) Lessons and challenges for measles control from unexpected large outbreak, Malawi. Emerg Infect Dis 19:202-209. doi:10.3201/eid1902.120301

Morbidity and Mortality Weekly Report (2011) Increased transmission and outbreaks of measles, European Region, 2011. Wkly Epidemiol Rec 86:559-564

Moss WJ, Griffin DE (2006) Global measles elimination. Nat Rev Microbiol 4:900-908. doi:10.1038/nrmicro1550

Parent du Châtelet I, Antona D, Freymuth F et al (2010) Spotlight on measles 2010: update on the ongoing measles outbreak in France, 2008-2010. Euro Surveill 15:2008-2010

Parker A, Staggs W (2006) Implications of a 2005 measles outbreak in Indiana for sustained elimination of measles in the United States. N Engl J Med 355:447-455

Roggendorf H, Mankertz A, Kundt R, Roggendorf M (2010) Spotlight on measles 2010: measles outbreak in a mainly unvaccinated community in Essen, Germany, March-June 2010. Euro Surveill 15:9-11

Scott S, Cumberland P, Shulman CE et al (2005) Neonatal measles immunity in rural Kenya: the influence of HIV and placental malaria infections on placental transfer of antibodies and levels of antibody in maternal and cord serum samples. J Infect Dis 191:1854-1860. doi:10.1086/429963

Shibeshi ME, Masresha BG, Smit SB et al (2014) Measles resurgence in southern Africa: challenges to measles elimination. Vaccine 32: 1798-1807. doi:10.1016/j.vaccine.2014.01.089

Siedler A, Mankertz A, Feil F et al (2011) Closer to the goal: efforts in measles elimination in Germany 2010. J Infect Dis 204(Suppl): S373-S380. doi:10.1093/infdis/jir068 
Sugerman DE, Barskey AE, Delea MG et al (2010) Measles outbreak in a highly vaccinated population, San Diego, 2008: role of the intentionally undervaccinated. Pediatrics 125:747-755. doi:10.1542/ peds. 2009-1653

van Treeck U (2006) Measles outbreak in Germany: over 1000 cases now reported in Nordrhein Westfalen. Euro Surveill 11:11-13

Waaijenborg S, Hahné SJM, Mollema L, Smits GP, Berbers GAM, van der Klis FRM, de Melker HE, Wallinga J (2013) Waning of maternal antibodies against measles, mumps, rubella, and varicella in communities with contrasting vaccination coverage. J Infect Dis 208:10 16. doi:10.1093/infdis/jit143

WHO (2012) Global measles and rubella. WHO Libr. Cat. Data Wolfson LJ, Grais RF, Luquero FJ et al (2009) Estimates of measles case fatality ratios: a comprehensive review of communitybased studies. Int J Epidemiol 38:192. doi:10.3201/eid1902. 120301 ples 